\title{
Early experience of laparoscopic varicocelectomy in College of Medical Sciences, Teaching Hospital, Bhartpur, Nepal
}

\author{
A.Agarwal ${ }^{1}$, G. Sigdel ${ }^{1}$, N.Lamichhane ${ }^{1}$, W.K.Belokar ${ }^{2}$ \\ ${ }^{1}$ Mch Resident ${ }^{2}$ Professor and HOD, Department of Urology, College of Medical Sciences and \\ Teaching Hospital, Bharatpur, Nepal
}

\begin{abstract}
Varicocele is dilatation of pampiniform plexus of veins in the scrotum. Laparoscopic varicocelectomy is gaining popularity since its introduction in 1990s. We present our experience of laparoscopic varicocelectomy in College of Medical sciences, Teaching Hospital, Bharatpur, Nepal.

Ten patients underwent laparoscopic varicocelectomy between Jan 2009 to July 2010. The mean age of patients was 25.7 (range, 14 to 38 ) years. All varicoceles were detected clinically and confirmed by scrotal color Doppler ultrasonography. The varicocele was left sided in $6(60 \%)$ and bilateral in 4 cases(40\%). The surgical indications were scrotal pain and discomfort in 8, infertility in 1, and both in 1. Mean operation time was $50 \pm 22$ minutes (range, 35-110). Following operation 6 patients were symptom free immediately and remaining 2 patients also became asymptomatic on 15 days followup. Both patients of infertility, lost follow up, post operatively. Neither recurrent varicocele nor hydrocele was detected with a mean follow-up of 13 months. All patients were discharged within 24 hours postoperatively. Every attempt was made to preserve spermatic artery and lymphatic vessel. The procedure is safe and effective.
\end{abstract}

\section{Key words: Infertility, laparoscopic, scrotal pain, varicocelectomy.}

\section{Introduction}

Varicocele is considered as one of the important causes of male infertility and prepubertal testicular hypotrophy. 1 The incidence of varicocele in the general population and infertile population is about $18 \%$ and $35 \%$, respectively.2,3 Treatment options include radiology guided sclerotherapy, surgical high or low ligation of the internal spermatic veins and recently, laparoscopic varicocelectomy. Laparoscopic varicocelectomy was introduced in early 1990s as an alternative to Palomo operation, since then the procedure has become more popular with almost the

Correspondence:A.Agarwal

E-mail:draaman@gmail.com same result as Palomo procedure. It can be done using intraperitoneal, pre-peritoneal and lumbotomy approach. Various laparoscopic varix ligation techniques have been described in the literature, like electrocautery, endo-clips, suture ligature, bipolar vessel sealer, etc. ${ }^{4,5}$ We report our preliminary results of a laparoscopic varicocelectomy using endo-clips through transperitoneal approach.

\section{Materials and methods}

The medical records of all patients who underwent laparoscopic varicocelectomy January 2009 and July 
A.Agarwal et al. Early experience of laparoscopic varicocelectomy in.

2010 in College of Medical sciences, Teaching Hospital, Bharatpur, Nepal, were analyzed. All varicoceles were detected clinically by physical examination and were confirmed by color doppler ultrasonography. The varicocele was classified as grade I when an impulse but not venous tortuosity appeared with Valsalva's maneuver, as grade II when a palpable tortuosity was evident through the skin with impulse on Valsalva's maneuver during the examination, and as grade III when a palpable torturosity was present without Valsalva's maneuver. Patients with grade II varicocele with symptoms and Grade III varicocele were subjected to laparoscopic varicocelectomy.All varicoceles were treated by transperitoneal laparoscopy(fig-1). Under general anesthesia, the patient was placed in the Tredelenburg position. Pneumoperitoneum was established using a Veress needle with an insufflating pressure between 10 and $12 \mathrm{mmHg}$. Then,10-mm,30 degree laparoscope was inserted through a 10-mm umbilical port. Next, a pair of 5-mm port were made between umbilicus and pubic symphysis and mid-point of spino-umblical line respectively(fig-2). An additional $5 \mathrm{~mm}$ ports at opposite spino-umblical line was made in bilateral varicocele cases. Diagnostic laparoscopy followed by opening of peritoneum over dilated vessels was done(fig-1). Laparoscopically the internal spermatic veins were confirmed as engorged vessels containing blood when the patient was placed in the reverseTrendelenberg's position or when the inguinal spermatic cord was squeezed in the cephalic direction intraoperatively. The internal spermatic artery was identified as a pulsatile vessel and this was possible in $90 \%$ of our cases. The lymphatic vessels were identified as vessels containing clear serous fluid without engorgement, in the above procedures. All collateral internal spermatic veins were identified, separated, clipped, and transected(fig-2). Every effort was made to save lymphatic vessels and artery but in 1 case mass clipping of veins and artery had to be done due to bleeding. The exposed peritoneum was closed intracorporeally with 3-0 vicryl suture(fig-2). No drain was kept. All patients were discharged on 1st post operative day. After operation, patients were followedup by physical examination and scrotal color Doppler ultrasonography. Three samples of semen for analysis were taken preoperatively in infertility cases.

\section{Results}

During the period between January 2009 and July 2010, 10 patients underwent laparoscopic varicocelectomy. The mean patient age was 25.7 (range, 14 to 38 ) years. All varicoceles were detected clinically and confirmed by scrotal color Doppler ultrasonography. The varicocele was left sided in 6 (60\%) and was bilateral in $4(40 \%)$. The surgical indications were scrotal pain and discomfort in 8 , infertility in 1, and both pain/discomfort and infertility in 1. Mean operation time was $50 \pm 22$ (range, 35110) minutes. The mean operation times for unilateral and bilateral varicocelectomies were 37 and 86 minutes, respectively. The mean number of clipped collateral veins was 2.5 (range, 1-5). All procedures were completed laparocopically without conversion. However, In 1 case mass clipping of dilated vessels were done due to intra-operative bleeding. . Every attempt was made to preserve spermatic artery and lymphatic vessel. No late procedure- related complications were noted. Following operation 6 patients were symptom free immediately and remaining 2 patients also became asymptomatic on 15 days followup. Both cases of infertility were lost to follow 
Journal of College of Medical Sciences-Nepal, 2012, Vol-8, No-2

up. Neither recurrence of varicocele nor hydrocele was detected with a mean follow-up of 13 months. No testicular atrophy was noted by scrotal ultrasound after the procedure. The cosmetic results of the laparoscopic trocar wounds were excellent. All patients were discharged within 24 hours postoperatively The procedure is safe and effective.

\section{Fig 1: Dilated pampiniform plexus and} mobilization of dilated veins laparoscopically.

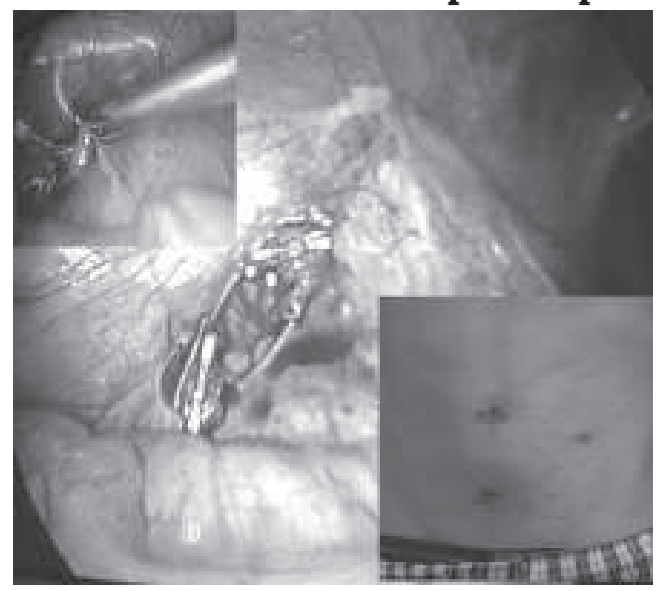

Fig 2: Clipping, transaction and closure of peritoneum with post operative port site scar mark.

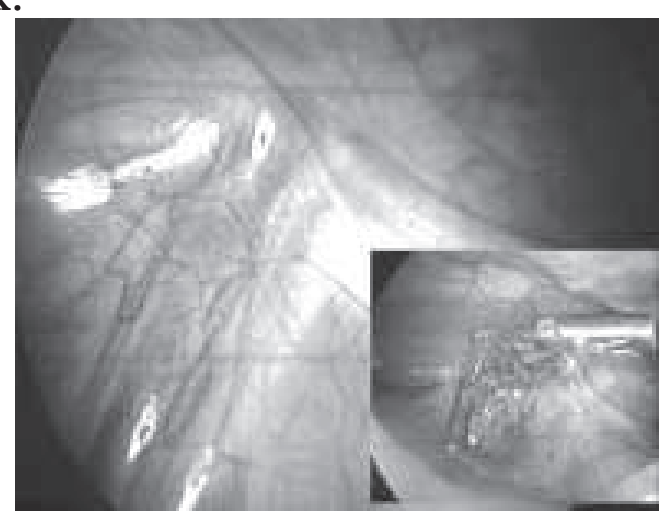

\section{Discussion}

Various surgical and radiological guided sclero therapy techniques have been used to manage varicoceles with variable success and complication rates. With advancements in laparoscopic instruments and techniques, a laparoscopic varicocelectomy has recently become one of the most commonly used techniques. ${ }^{6}$ However, laparoscopic surgery is considered to be a more-expensive and timeconsuming procedure when compared to traditional open techniques.Our preliminary results revealed that laparoscopic varicocelectomy is safe as well as effective in improving varicocele-related symptoms. We could not assess improvement in semen parameter as both of our infertile patients did not turn up for follow up. Literature suggest that it helps in improving semen quality. Fourty percent of our cases had bilateral disease, and the time-consuming procedures of skin incision and closure was eliminated with the laparoscopic varicocelectomy. Some of the reported advantages of a laparoscopic varicocelectomy are as follows. First, an aberrant course (bifurcation) of the internal spermatic veins, which can possibly be missed by a traditional open high ligation, can be identified and definitively treated. Second, an incidental inguinal hernia can be repaired at the same time. Third, bowel adhesions over the course of the internal spermatic vessels, which might have something to do with the patient's lower abdominal discomfort, can be released during the operation. We did not encounter either hernia or adhesions in any of our cases. Drawbacks of this technique are that it is technically more demanding and requires a relatively longer operative time. ${ }^{7,8}$ Preservation of the artery and lymphatic vessel eliminate the risk of testicular ischemia and hydrocele formation. ${ }^{7}$ Neither postoperative hydrocele formation nor varicocele recurrence was documented in our series. Testicular atrophy following varicocelectomy is a risk associated with ligation of the testicular artery. ${ }^{9}{ }^{10}$ None of our cases showed atrophy in follow up USG of scrotum. The traditional mass high ligation of the internal spermatic cord carries a higher risk of persistent hydrocele postoperatively when compared 
A.Agarwal et al. Early experience of laparoscopic varicocelectomy in..

to testicular artery-sparing procedures. ${ }^{6,11-12}$ In addition,

division of the lymphatic channels is associated with postoperative testicular edema and reduced testicular function. ${ }^{11}$ Mass ligation of the internal spermatic vessels potentially carries the risk of testicular venous infarction ${ }^{12}$ and higher incidence of postoperative hydrocele due to lymphatic ligation. We preferred to clip all internal spermatic veins and tried to preserve all arterial and lymphatic vessels to minimize possible complications from arterial and lymphatic ligation. Our experience confirmed that good postoperative results and minimal complications can be achieved using laparoscopic technologies.A high rate of bilateral varicoceles $(67 \%-73 \%)$ has been documented by others ${ }^{13}$ but our series showed only $40 \%$ cases with bilateral varicocele. Controversy over whether a subclinical small right varicocele associated with a left clinical palpable varicocele should be ligated, still exists. In a prospective study, a bilateral varicocelectomy achieved significantly better semen quality improvement than a unilateral varicocelectomy in infertile men with bilateral varicoceles. ${ }^{14}$

\section{Conclusion}

Our results reveal that laparoscopic varicocelectomy is a safe and effective procedure in improving varicocele related symptoms. Larger case series and proper follow up is required to comment its role in infertility cases though literature supports its role in improving semen quality. Preservation of the internal spermatic artery and lymphatic channels minimize postvaricocelectomy-related complications and its recurrence.

\section{References}

1. E.J. Kass, C. Reitelman. Adolescent varicocele. Urol Clin Am 1995;22:151-9.

2. J.P. Jarow, M. Coburn, M. Sigman. Incidence of varicoceles in men with primary and secondary infertility. Urology 1996;47:73-6.

3. R.B. Meacham, R.R. Townsend, E. Rademacher, et al. The incidence of varicoceles in the general population when evaluated by physical exam, gray scale sonography and color Doppler sonography. J Urol 1994;151:1535-8.

4. Sanchez de Badajoz E, Jimenez Garrido A. Microlaparoscopic varicocelectomy. Arch Esp Urol 2002;55:659-64.

5. N. Sun, T.T. Cheung, P.L. Khong, Varicocele: laparoscopic clipping and color Doppler follow-up. J Pediatr Surg 2001;36:1704-7.

6. C. Esposito, G.L. Monguzzi, M.A. Gonzalez-Sabin, et al. Laparoscopic treatment of pediatric varicocele: a multicenter study of the Italian Society of Video Surgery in Infancy. J Urol 2002;163:1944-6.

7. K. Itoh, Y. Suzuki, H. Yazawa, et al. Results and complications of laparoscopic Palomo varicocelecctomy. Arch Androl 2003;49:107-10.

8. I. Kbaier, M.Y. Binous, F. Attyaoui et al. Laparoscopic spermatic vessel ligation in the treatment of varicocele: report of 129 procedures. Ann Urol 2002;36:329-33.

9. R.A. MacMahon, M.C. O'Brien, L.J. Cussen. The use of microsurgery in the treatment of the undescended testis. J Ped Surg 1976;11:521-6. 
Journal of College of Medical Sciences-Nepal, 2012, Vol-8, No-2

10. S.J. Silber. Microsurgical aspects of varicocele. Fertil Steril 1979;31:230-2.

11. R. Kocvara, J. Dolezal, R. Hampl, et al. Division of lymphatic vessels at varicocelectomy leads to testicular edema and decline in testicular function according to the LH-RH analogue stimulation test. Eur Urol 2003;43:430-5.
12. R.D. Amelar. Early and late complications of inguinal varicocelectomy. J Urol 2003;170:366-9.

13. S. Cayan, F. Erdemir, I. Ozbey. Can varicocelectomy significantly change the way couples use assisted reproductive technologies? J Urol 2002;167:1749-52.

14. D. Scherr, M. Goldstein. Comparison of bilateral versus unilateral varicocelectomy in men with palpable bilateral varicoceles. J Urol 1999;162:85-8. 